\title{
Analyses on the Equilibrium Model of Financing Game -Based on Block Chain Technology
}

\author{
Qingyuan feng \\ Canvard College, Beijing Technology and Business University, Beijing, \\ Work address: No.1, Songzhuang South Road, Tongzhou, Beijing, China (code: 101118) \\ Mailing address: Yitong community, Shayang Road, Shahe, Changping, Beijing, China (code: 102206) \\ Email: hwhqy@163.com
}

\begin{abstract}
China's small and micro enterprises have made and are making indispensable contributions to promote the development of the national economy, increase employment and maintain social stability. However, the traditional financing channel is single, the financing cost is high, and the financing difficulties greatly restrict many enterprises to further develop and grow. Under the background of rapid development of financial science and technology represented by block chain, big data, cloud computing, artificial intelligence and so on, financial models are constantly being innovated, and Internet financing models are increasingly becoming an important and effective way to raise funds for small and micro enterprises in the future. Using dynamic game theory tools, this paper makes model analysis on financing modes based on block chain technology, and draws a conclusion that financing modes that improve information asymmetry based on block chain can better alleviate the financing difficulties of small and micro enterprises. Finally, it points out the risks existing in the application of block chain technology in the credit field at present, and puts forward some suggestions, hoping to promote the healthy development of financial science and technology industry including Internet financing industry.
\end{abstract}

Keywords: financing, game theory, block chain

\section{Introduction}

Since the reform and opening up, all kinds of enterprises in our country have made great progress and played an important role in promoting economic development, increasing employment and maintaining social stability. In particular, some small and medium-sized microenterprises that have emerged in recent years under the encouragement and support of policies have made great contributions in solving employment problems and promoting economic development. However, due to the current development of China's financial industry and its own actual situation, small and medium-sized microenterprises generally have the problems of "single financing channel, difficult financing and high financing cost"[1]. In a financing research project presided over by the department responsible for the development of small and medium-sized micro-enterprises of the National Development and Reform Commission, it can be seen that over $50 \%$ of the surveyed enterprises believe that their development is constrained by insufficient funds. In the past three years, about $48 \%$ of the enterprises have not been able to successfully raise funds, while another $30 \%$ of the enterprises have only obtained a small amount of financing despite having successfully raised funds. From the point of view of small and medium-sized enterprises themselves, the lack of a standardized and sound management system, highly opaque information, and difficulty in providing sufficient mortgage or guarantee in financing make traditional financial institutions unwilling to finance them due to worries about weak economic strength, high risk of default, poor business continuity and high possibility of bankruptcy[2]. In terms of banks, the current banking system is dominated by large state-owned banks and discriminates against enterprises inside and outside the system. From a policy perspective, the credit system for small, medium and micro enterprises has not yet been established, the credit guarantee system is not perfect, and the information sharing mechanism among commercial banks has not taken shape. A variety of internal and external factors have caused the traditional direct financing channels of small and micro enterprises to be blocked, the difficulties in bank loans and higher financing costs, seriously inhibiting the development of such enterprises and further affecting social innovation, economic vitality and sustainable development of the national economy[3].

\section{Technical Characteristics of Block Chain}

With the continuous development of emerging technologies represented by block chain, artificial intelligence, cloud computing and big data, science and technology+financial enterprises are rapidly rising. With their advantages and characteristics of user behavior value, market selection, cost intensification, resource opening 
and channel autonomy, they are constantly creating new financial modes, such as technology and finance modes such as third-party payment, big data finance, crowdfunding, information-based financial institutions and Internet financial portals, which have brought subversive impacts to many fields of traditional financial industry and have made breakthroughs to the core fields of financial industry[4]. Among these emerging technologies, block chain technology, as a subversive technology, also has the ability to trigger a new round of technological innovation and industrial revolution. At present, many countries have begun to gradually realize the strategic significance and commercial value of block chain technology, and at the same time, they have begun to conduct comprehensive research and exploration in this technical field.

\subsection{De-centralization}

Compared with the traditional information technology, the core feature of the block chain is "de-centralization", which is the basis for the rapid development of block chain technology. In the traditional data processing environment, the star-shaped architecture mode is generally adopted, the core processing module is in the central position of the whole application architecture, and a large number of clients are connected to the central server through a network system to form a radial mesh structure. In this structure, whether the core processing module can maintain a continuous, stable and safe operation directly affects the operation effect of the whole system. If the central server is injected with illegal malicious code or attacked, it will bring great security threats to the system and users. However, the block chain technology has abandoned the traditional mode and adopted a completely different idea. It distributes the processing logic and storage to all nodes in the network. Each node has equal status[5]. They jointly complete the data recording, confirmation and verification. Each network node is responsible for the transaction processing, storage and data verification. The processing process is also completely transparent in the whole chain. In this non-core architecture mode, single-point or multi-point attacks will not pose a threat to system security, nor will the failure of one or several nodes cause the whole system to be abnormal, let alone crash (see Figure 1).
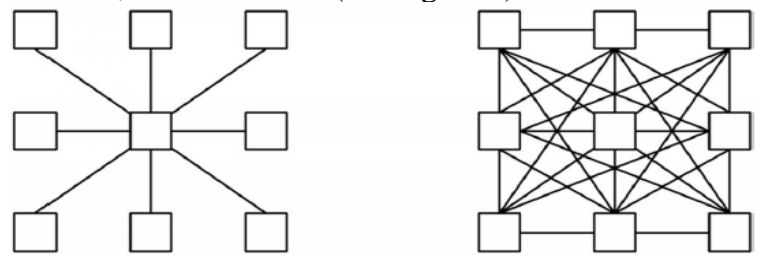

Fig 1 illustration of centralization and decentralization

\subsection{Non-Tampering}

The data stored in the block chain node is not tamperable, and it mainly uses hash algorithm to ensure the security of the data. The realization idea of this algorithm is to accept a piece of unencrypted data information, and use an irreversible algorithm to generate a piece of hash information with short length and fixed number of bits. Since hash algorithm itself has the feature of irreversible operation, this means that no one can infer any original information related to the original text from the content of the output hash value[6]. Any change in input information, even if it is only a single digit change, will cause obvious changes in the processing results, so the illegal modification of node information in the block chain can be fundamentally solved.

\subsection{Anonymity}

The data recorded in the block chain adopts a "non-realname" method, each operator is anonymous, and at the same time the operation information of each non-realname user is transparent, so we can see the real data of each transaction in the block chain, but it cannot correspond to a specific operator and has desensitization characteristics[7]. Of course, anonymity is a double-edged sword. While protecting users' privacy, it may also bring some negative effects, such as providing an umbrella for illegal and criminal acts. In this case, it is difficult for law enforcement agencies to find out the culprit. Therefore, in practical application, how to avoid its negative effects while giving full play to the advantages of block chain anonymity should be one of the focuses of future research.

\section{ANALYSIS OF FINANCING GAME UNDER BLOCK CHAIN TECHNOLOGY ENVIRONMENT}

The development of Internet finance helps to alleviate the financing difficulties of small and micro enterprises. However, while solving this difficult and expensive problem, the financial service real economy is facing another big challenge-the information asymmetry between the supply and demand sides[8]. The above characteristics of the block chain technology can better improve the information asymmetry between the supply and demand sides of the small and medium-sized micro-enterprises and their supply chains, and truly realize the point-to-point lending of disintermediation. Based on the block chain technology, this paper attempts to use game theory tools to make theoretical reasoning analysis on the game between investors and small and medium-sized micro-enterprises on peer-to-peer lending. In order to simplify the game analysis, the following assumptions are made:

First, investors and small and medium-sized microenterprises are rational economic men.

Second, there is no information asymmetry between investors and SMEs. 
The third is a good credit constraint mechanism. In the block chain technology environment, information is contained in each block with password protection. At this time, the absolute cost of counterfeiting information will reach a very high level, which is almost impossible under the current technology conditions[9]. The cost of counterfeiting information will be very high relative to the total amount of funds raised, and financiers will also pay an extremely high price after default. So the possibility of default is zero.

Set the investment volume as I, the total return after the success of the project is $\mathrm{R}(\mathrm{R}>\mathrm{I})$, and the investor's return on capital is $r$. Relative default cost $\mathrm{B}>0$. Financiers will also pay an extremely high price after default, and the relative default cost after the sum of the pre-fake cost and the post-default cost is set to $\mathrm{C}>0$.

table 1 Block Chain Credit Game Model

\begin{tabular}{|c|c|c|c|}
\hline \multirow{4}{*}{ Investors } & \multicolumn{3}{|c|}{ financiers } \\
\cline { 2 - 4 } & & Default & No Default \\
\cline { 2 - 4 } & Investment & $-\mathrm{I},-\mathrm{C}$ & $\mathrm{Ir}, \mathrm{R}-\mathrm{I}$ \\
\cline { 2 - 4 } & No Investment & $0,-\mathrm{B}$ & 0,0 \\
\hline
\end{tabular}

According to the game model, there is only one Nash equilibrium (investment, no default). The Nash equilibrium can maximize social utility. The financier will not forge information in advance in order to obtain funds, there is no adverse selection problem, and there will be no moral hazard problem after financing. The fund user will make maximum use of the obtained funds and reduce the agency cost of both parties to the greatest extent. At this time, investors can also invest with confidence[10].

From the conclusion of the model, it can be seen that the core of the introduction of block chain technology into the credit field lies in more transparent information. The cost of forging information and defaulting will increase significantly, so that financiers have no motivation to forge and default. To a large extent, this will not only change the current financing status of individuals and enterprises, but also have a positive impact on investors[11]. Investors do not have to worry about being cheated or defaulted afterwards (even if there is a default afterwards, it is a very small probability). It can be seen that the block chain financial model will form a financing market for investors and financiers to achieve a win-win situation. The introduction of the block chain will not only have a positive impact on the financing mode, but will definitely change the investment and financing behavior of both participants.

\section{APPLICATION RISKS OF BLOCK CHAIN TECHNOLOGY}

As a distributed computing technology, block chain has the characteristics of de-centralization, information tamper-proofing, openness and transparency, traceability, verifiability, etc. Applying block chain technology to the credit industry can effectively make up for the deficiencies of the traditional third-party credit system. The use of block chain technology can effectively alleviate adverse selection and moral hazard problems caused by information asymmetry, thus reducing the credit default rate[12]. Embedding intelligent contract, one of the characteristic technologies of block chain, into intelligent loans can reduce the cost of information acquisition and labor, and improve the efficiency of business processing. Although the application of block chain technology in the credit field has many advantages, at present block chain is still in the initial stage of development, lacking relevant specific operation experience and empirical research, and there are many risks[13]. Therefore, in view of the possible problems in its credit application, this paper puts forward the following suggestions:

One is the technical level of the block chain, which mainly includes three major problems: redundant storage, data security accidents and poor correctability. With the passage of time, the amount of data storage on the block chain increases until it exceeds the carrying capacity of the existing computer storage system. At the same time, the maintenance cost for the block chain system data also increases. In the early stage of block chain application, if there are too few participating nodes, hackers can easily control more than $51 \%$ of the nodes, which will easily lead to system data security accidents[14]. Once confirmed, the data on the block chain cannot be tampered with, which makes it impossible to correct the wrong data in time. To solve these problems, it is necessary to intensify the research on block chain technology and increase the research investment in block chain storage, asymmetric encryption technology, network security technology, etc.

Second, the application standards of block chain technology are not uniform. At present, there is no uniform standard for the application of block chain technology in the credit field, and how to realize the effective integration of traditional credit system and block chain system is also a big problem. Therefore, it is urgent to strengthen the communication and coordination of the application of the block chain and jointly formulate the industry standards in the form of alliance chain. Considering the application of the combination of block chain technology and big data, application experiments are carried out to gradually transition the traditional credit system to the advanced block chain system.

The third is supervision. The application and popularization of a new technology will inevitably involve risks. At present, the block chain technology is still in the initial stage of development. The regulatory authorities have not fully and deeply understood the block chain technology, the regulatory attitude is not clear, and the relevant regulatory laws and regulations are lagging behind[15]. In this regard, the regulatory authorities should track and understand the application and development of block chain technology in real time, properly supervise and formulate laws and regulations conducive to its healthy application and development. At the same time, credit industry departments should actively cooperate, actively strengthen communication and coordination with regulatory authorities, and carry out application 
[5] Babbitt, what can and cannot be done by the block chain to solve the difficulties of credit business, Business news, 2018( 1):35-36

[6] Long Tingting, Application of Block Chain Technology in Business Model Innovation of Commercial Banks, Journal of Liaoning Economic Management Cadre Institute, 2018( 1):14-16

studied the introduction of block chain technology into the credit investment and financing market, which will fundamentally alleviate the credit difficulties encountered by small and micro enterprises in their operations, and analyzed the traditional bank credit and the financing model based on block chain technology by game models respectively, pointed out the risks existing in the application of block chain and put forward suggestions.

Block chain technology is still in continuous development and improvement. There are certainly many technical problems and many difficult problems to be solved in the block chain financial model. Although the author is not entirely sure whether the introduction of the block chain into the financial market will bring about a complete change in finance as described in this article, it is undeniable that the block chain financial model will become a useful supplement to China's gold market in the future. It can be predicted that the combination of block chain technology and traditional credit industry will be an important direction for future financial development. Therefore, the credit industry must fully participate in the research and application of block chain technology as soon as possible. While continuously improving its adaptability and innovation ability, it will also provide some help for the application and development of block chain technology, and at the same time, it will also provide a wider and more convenient investment and financing choice channel for small and micro enterprises and ordinary people in China.

\section{REFERENCES}

[1] Jiang Xiaowei, Research on Financing Problems and Development Strategies of Small and Mediumsized Enterprise Clusters, Research on Commercial Economy, 2019(17):161-164

[2] Wang lihui, empirical analysis of financial science and technology and financing of small and mediumsized enterprises, technical economy and management research, 2017(2):93-97

[3] Li Zhongfei, Huang Jinbo, Dynamic Game Analysis of Financing Difficulties of Small and Micro Enterprises in China, East China Economic Management, 2016 (2): 1-8

[4] Annual Comprehensive Submersion, Payment for Navigation, Agreement and Path between Block Chain Technology and Inclusive Finance, International Finance, 2018(8):76-80
[7] Xie Ping, Zou Chuanwei, Internet Financial Model Research [J], Financial Research, 2012 (12): 11-22

[8] EYAL I, GENCER A E, SIRER E G, et al. Bitcoinng: a scalable blockchain protocol[C],USENIX Symposium on Networked Systems Design and mplementation. USENIX, 2016: 45-59

[9] SWAN M. Blockchain: blueprint for a new economy[M],SWAN M.Blockchain: Blueprint for A New Economy. Sebastopol: O'Reilly,2015

[10] Swan, M., Blockchain: blueprint for a new economy[M]. Sebastopol: O'Reilly Media, 2015

[11] GARAY J A, KIAYIAS A, LEONARDOS N, et al. Bootstrapping the blockchain, with applications to consensus and fast PKI setup[C],IACR International Workshop on Public Key Cryptography.Springer, 2018: 465-495

[12] KIAYIAS A, RUSSELL A, DAVID B, et al. Ouroboros: a provably secure proof-of-stake blockchain protocol[C],International Cryptology Conference. 2017: $357-388$

[13] STOSIC D, STOSIC D, LUDERMIR T B, et al. Nonextensive triplets in ryptocurrency exchanges[J]. Physica A Statistical Mechanics \& Its Applications, 2018(505): 1069-1074.

[14] Xu Duo, Problems and Countermeasures in Developing Digital Inclusive Finance in China, Modernization of Shopping Mall, 2018 (08): 123-124

[15] Yang Jun, Financial Lever, Signaling Game and Credit Risk Identification, Financial Research, 2004 (02): 71-78. 